\title{
Physiologic cold shock of Moraxella catarrhalis affects the expression of genes involved in the iron acquisition, serum resistance and immune evasion
}

Violeta Spaniol ${ }^{1}$, Rolf Troller ${ }^{1}$, André Schaller ${ }^{2}$ and Christoph Aebi ${ }^{1,3^{*}}$

\begin{abstract}
Background: Moraxella catarrhalis, a major nasopharyngeal pathogen of the human respiratory tract, is exposed to rapid downshifts of environmental temperature when humans breathe cold air. It was previously shown that the prevalence of pharyngeal colonization and respiratory tract infections caused by $M$. catarrhalis are greatest in winter. The aim of this study was to investigate how M. catarrhalis uses the physiologic exposure to cold air to upregulate pivotal survival systems in the pharynx that may contribute to $M$. catarrhalis virulence.

Results: A $26^{\circ} \mathrm{C}$ cold shock induces the expression of genes involved in transferrin and lactoferrin acquisition, and enhances binding of these proteins on the surface of $M$. catarrhalis. Exposure of $M$. catarrhalis to $26^{\circ} \mathrm{C}$ upregulates the expression of UspA2, a major outer membrane protein involved in serum resistance, leading to improved binding of vitronectin which neutralizes the lethal effect of human complement. In contrast, cold shock decreases the expression of Hemagglutinin, a major adhesin, which mediates B cell response, and reduces immunoglobulin D-binding on the surface of $M$. catarrhalis.

Conclusion: Cold shock of $M$. catarrhalis induces the expression of genes involved in iron acquisition, serum resistance and immune evasion. Thus, cold shock at a physiologically relevant temperature of $26^{\circ} \mathrm{C}$ induces in $M$. catarrhalis a complex of adaptive mechanisms that enables the bacterium to target their host cellular receptors or soluble effectors and may contribute to enhanced growth, colonization and virulence.
\end{abstract}

\section{Background}

Moraxella catarrhalis colonizes the mucosal surface of the human nasopharynx and is a major cause of acute otitis media in children and of exacerbations of chronic obstructive pulmonary disease in adults [1,2]. Clinical studies have revealed that the prevalence of pharyngeal colonization and respiratory tract infections caused by $M$. catarrhalis displays seasonal variation and increases in winter [3-6]. Because breathing cold air (e.g., $-1^{\circ} \mathrm{C}$ at $10-20 \mathrm{l} / \mathrm{min}$ ) reduces the nasopharyngeal temperature from $34^{\circ} \mathrm{C}$ at room temperature to $\sim 26^{\circ} \mathrm{C}$ within several minutes and for extended periods of time [7], the human nasopharyngeal flora is repeatedly exposed to

\footnotetext{
* Correspondence: christoph.aebi@insel.ch

IInstitute for Infectious Diseases, University of Bern, CH-3010 Bern, Switzerland

Full list of author information is available at the end of the article
}

rapid downshifts of environmental temperature. In addition to viral infections that pave the way for subsequent secondary bacterial infections [8], the rapid downshift of temperature induces adaptive events in the residential upper respiratory tract flora that may lead to the transition from asymptomatic colonization to bacterial secondary infection. Our previous findings established that a $26^{\circ} \mathrm{C}$ cold shock upregulates the expression of UspA1, a major adhesin and putative virulence factor of $M$. catarrhalis, and promotes $M$. catarrhalis adherence to upper respiratory tract cells via enhanced binding to fibronectin $[9,10]$. Exposure of M. catarrhalis to $26^{\circ} \mathrm{C}$ also increases the outer membrane protein (OMP)mediated release of the proinflammatory cytokine IL-8 in pharyngeal epithelial cells and reduces the expression of porin M35, which may affect the resistance to aminopenicillins $[10,11]$. 
Among the various putative virulence factors that have been identified to date, several other proteinaceous antigens including lactoferrin-binding proteins (LbpA/B), transferrin-binding proteins (TbpA/B), CopB, UspA2 and Hemagglutinin (Hag/MID) may be involved in the cold shock response and thus be important in adapting to and colonizing the human host. Iron is an essential nutrient for most bacteria and $M$. catarrhalis overcomes the host's restriction of free iron through the evolution of iron acquisition systems which enable it to use lactoferrin, transferrin, hemoglobin, and hemin as iron sources. The primary site of $M$. catarrhalis entry into the human host is the nasopharynx, where lactoferrin is the predominant source of iron. Therefore, efficient iron acquisition from lactoferrin is an important virulence factor for pathogenic bacteria. The surface protein CopB is involved in the ability of M.catarrhalis to acquire iron from human transferrin and lactoferrin and is also an important factor responsible for complement evasion $[12,13]$. UspA2, a major OMP of $M$. catarrhalis, binds vitronectin, a component of both plasma and the extracellular matrix, and confers serum resistance of $M$. catarrhalis [14]. Furthermore, the UspA2 is able to bind human C3 and C4bp protecting M. catarrhalis from complement-mediated killing $[15,16]$. The surface protein Hag/MID that acts as an adhesin and hemagglutinin, exhibits unique immunoglobulin (Ig) D-binding properties and binds to both soluble and membranebound IgD on B cells [17-19]. Our previous study demonstrated that exposure of $M$. catarrhalis to $26^{\circ} \mathrm{C}$ down-regulates hag mRNA expression [9], indicating a possible involvement of Hag in the cold shock response.

In the present study we investigated the effect of a $26^{\circ}$ $\mathrm{C}$ cold shock on the expression of genes involved in iron acquisition, serum resistance and immune evasion. Cold shock induced the expression of genes involved in transferrin/lactoferrin acquisition and enhanced binding of these proteins on the surface of $M$. catarrhalis. Exposure of $M$. catarrhalis to $26^{\circ} \mathrm{C}$ upregulated the expression of UspA2, a major OMP involved in serum resistance, leading to the improved vitronectin binding. In contrast, cold shock decreased the expression of Hag, a major adhesin mediating B cell response, and reduced IgD-binding on the surface of $M$. catarrhalis.

\section{Methods}

Bacterial strains and culture conditions

M. catarrhalis strain O35E, its isogenic tbpB (O35E. tbpB), uspA1 (O35E.uspA1), uspA2 (O35E.uspA2), hag (O35E.hag) and $l p x A$ (O35E.lpxA) mutants, and clinical isolates 300 and 415 have been described elsewhere $[9,20,21]$. Bacteria were cultured at $37^{\circ} \mathrm{C}$ and $200 \mathrm{rpm}$ in brain heart infusion (BHI) broth (Difco) or on BHI agar plates in an atmosphere containing $5 \% \mathrm{CO}_{2}$. Cold shock experiments were performed as described [9]. Bacteria were grown overnight at $37^{\circ} \mathrm{C}$, resuspended in fresh medium and grown to mid-logarithmic phase (optical density at $600 \mathrm{~nm}\left[\mathrm{OD}_{600}\right]$ of 0.3 ). Subsequently, bacteria were exposed to $26^{\circ} \mathrm{C}$ or $37^{\circ} \mathrm{C}$, respectively, for 3 hours (unless otherwise stated). The growth rates of $M$. catarhalis under iron depletion conditions were evaluated by culturing the bacteria in BHI containing $30 \mu \mathrm{M}$ desferioxamine (Desferal; Novartis).

\section{RNA methods}

RNA for mRNA expression analysis was isolated and used for complementary DNA (cDNA) synthesis as described elsewhere [9]. Generated cDNA was amplified by semi-quantitative polymerase chain reaction (PCR) using primers for $l b p B$ (5'-GCAAGGCGGTAGGGCAGAT-3', 5'-CCTGCTTTTTCGGCGGTGTC-3'), lbpA (5'-AACAACGCATTCACAGCACCGATT-3', 5'-GATA CCAAGACGAGCGGTGATG-3'), $t b p B$ (5'-CAAG CAGGCCGGTGGTATGG-3', 5'-GGTAAATGGGGT GAATGTGGTTGC-3'), $t b p A$ (5'-AAGGCGGAGGCAA CAGATAAGACA-3', 5'-AGAGCCAGATAATGCCCC AGAGC-3') and 16S ribosomal RNA [rRNA] (5'-AA GG TTTGATC(AC)TGG(CT)TCAG-3', 5'-CTTTACGC CCA(AG)T(AG)A(AT)TCCG-3').

Quantitative real-time PCR was performed in triplicate for both target and normalizer (16S rRNA) genes. Notemplate controls and RT-negative controls were included in each run. Primers and probes were purchased from Applied Biosystems. Primers for $t b p B$ were 5'-TCCTTTCACTTCGCTAAATCGGTTT-3', 5'-CCACACAAGATGCGGTCAAATATAAA-3', and TaqMan probe was 5'-(FAM)CCTTTGTTGGCAACATC-3'. Primers for uspA2 were 5'-GCCTTAGACACCAAAGTCAATGC-3', 5'-AAGCTGCCCTAAGTGGTCTATTC3', and TaqMan probe was 5'-(FAM)TGAAAACGGTATGGCTG-3'. Primers and probes for hag and 16S rRNA were used as described elsewhere $[9,22]$. Relative quantification of gene expression was performed using the comparative threshold method. The ratios obtained after normalization were expressed as folds of change compared with samples isolated from bacteria exposed for $1 \mathrm{~h}$ at $37^{\circ} \mathrm{C}$.

\section{Immunoblotting}

OM vesicles, composed of OMPs and lipooligosaccharide (LOS), from strain O35E exposed for $3 \mathrm{~h}$ to either $26^{\circ} \mathrm{C}$ or $37^{\circ} \mathrm{C}$ were prepared by the EDTA buffer method [23]. Samples were resolved by SDS-PAGE using a $7.5 \%$ polyacrylamide gel and transferred to polyvinylidene difluoride membranes (Millipore). Lactoferrin binding was detected using mouse anti-human lactoferrin monoclonal antibody (AbD Serotec) and horseradish peroxidase (HRP)-conjugated goat anti-mouse antibody 
(Sigma). IgA-binding was detected using human saliva samples as the primary antibody source and HRP-conjugated goat anti-human IgA (Sigma) as secondary antibody. Sampling of saliva from healthy volunteers was approved by the local ethics committee.

\section{Solid-phase lactoferrin binding assay}

Detection of lactoferrin binding to $M$. catarrhalis was performed as described elsewhere [24]. Equal amounts of strain $\mathrm{O} 35 \mathrm{E}$ grown at $26^{\circ} \mathrm{C}$ or $37^{\circ} \mathrm{C}$ for $3 \mathrm{~h}$ were spotted onto the nitrocellulose membranes. The blots were blocked in Tris-buffered saline $(50 \mathrm{mM}$ Tris buffer containing $0.1 \mathrm{M} \mathrm{NaCl}[\mathrm{pH} 7.0]$ ) containing $0.5 \%$ nonfat dry milk and incubated with human lactoferrin $(10 \mu \mathrm{g} /$ $\mathrm{mL}$ ), followed by a mouse anti-human lactoferrin antibody and developed by using horseradish peroxidaseconjugated goat anti-mouse antibody.

\section{Two-dimensional gel electrophoresis (2-DE)}

Analysis of OMPs spots of strain O35E was performed as described previously [25]. To identify the proteins indicated in Figure 1, the MALDI-TOF was used [25]. Protein concentration was determined using the 2-D QuantKit (Amersham). Differential analysis was performed using the ImageMaster 2D Platinum software version 5.0 (Amersham) for spot detection, quantification, matching and comparative analysis. The expression level was determined by the relative volume of each spot in the gel and expressed as \%Volume $(\% \mathrm{Vol}=($ spot volume $/ \Sigma$ volumes of all spots resolved in the gel)). This normalized spot volume takes into account variations due to protein loading and staining by considering the total volume over all the spots present in the gel. A collection of 6 gels ( 3 of each temperature) resulting from three independent experiments was analyzed. Spots detected by the program were matched between the three gel pairs. Variations in abundance were calculated as the ratio of average values of \%Vol between two temperatures. Only spots with a \% Vol variation ratio greater than 2 (with significance set at 2-fold change) in the ImageMaster 2D Platinum report were considered relevant.

\section{Treatment of $M$. catarrhalis with lactoferrin}

Treatment of $M$. catarrhalis with lactoferrin was performed as described elsewhere [26]. Strain O35E was grown to an $\mathrm{OD}_{600}$ of 0.5 , resuspended in assay solution containing $0.1 \%$ gelatine to a concentration of $10^{5} \mathrm{CFU} /$ $\mathrm{mL}$ prior to the addition of lactoferrin $(1 \mathrm{mg} / \mathrm{mL}$, Sigma). Samples were incubated at $37^{\circ} \mathrm{C}$ for 1 and $3 \mathrm{~h}$ followed by plating on BHI agar to determine viability.

\section{Flow cytometry}

Bacteria were exposed to $26^{\circ} \mathrm{C}$ or $37^{\circ} \mathrm{C}$ for $3 \mathrm{~h}$. The $\mathrm{OD}_{600}$ was adjusted to 0.2 , the $200-\mu \mathrm{L}$ aliquots were washed in PBS-1\% BSA, and incubated with $1 \mu \mathrm{g} / \mathrm{mL}$ of lactoferrin or with $1 \mu \mathrm{g}$ of vitronectin (Millipore) for 1 h. To assess the ability of $M$. catarrhalis to bind salivary lactoferrin, bacteria were preincubated with saliva samples (1:20 dilution) from healthy adults. Bacteria were incubated with mouse anti-human lactoferrin monoclonal antibody (AbD Serotec) or mouse anti-human vitronectin monoclonal antibody (Quidel) followed by incubation with Alexa 488-conjugated goat anti-mouse antibody (Invitrogen) and analyzed on a FACScan cytometer using CellQuest software (version 4.2; BD Bioscience). Anti-human lactoferrin or vitronectin antibodies and Alexa 488-conjugated anti-mouse antibody were added separately as negative controls.

Binding of transferrin to M. catarrhalis was analyzed using fluorescein isothiocyanate (FITC)-conjugated human transferrin $(0.1 \mu \mathrm{g} / \mathrm{mL}$, Jackson Immunoresearch).

The ability of $M$. catarrhalis to bind human IgD was analyzed as described elsewhere [27]. Strain O35E, Hagdeficient mutant (O35E.hag), LOS-deficient mutant (O35E.lp $x A$ ) and clinical isolate 300 were exposed to $26^{\circ}$ $\mathrm{C}$ or $37^{\circ} \mathrm{C}$ for $3 \mathrm{~h}$, harvested, and incubated with $50 \%$ of pooled normal human serum (NHS) as a source of IgD, followed by a FITC-conjugated rabbit anti-human IgD polyclonal antibody (Dako).

The expression of UspA1/A2 and CopB was analyzed using the uspA1/A2-specific $17 \mathrm{C} 7$ and the $\operatorname{cop} B$-specific 10F3 (1:20) mouse monoclonal antibodies.

\section{Statistical analysis}

Data were expressed as mean \pm 1 standard deviation (SD). Differences between groups were analyzed by oneway analysis of variance with a Bonferroni posttest using Prism software (version 5.01; GraphPad). $P<0.05$ was defined as statistically significant.

\section{Results}

OM proteome analysis following cold shock in $M$. catarrhalis

To assess cold shock-induced changes in the OM proteome of $M$. catarrhalis, 2-DE analysis was used. OMPs were isolated from a culture of $M$. catarrhalis strain O35E, which was exposed to a 3-hour cold shock at $26^{\circ}$ $\mathrm{C}$ or to continuous growth at $37^{\circ} \mathrm{C}$. A collection of 6 gels (3 of each temperature) resulting from three independent experiments was analyzed. Three OMPs $(\sim 75$ $\mathrm{kDa}, \mathrm{pI} 9 ; 50 \mathrm{kDa}, \mathrm{pI} 7$; and $14 \mathrm{kDa}, \mathrm{pI} 8$ ) were found to be differentially (a greater than twofold change) regulated in response to a $26^{\circ} \mathrm{C}$ cold shock (Figure 1). Among these proteins, two spots (75 and $15 \mathrm{kDa}$ ) were upregulated and one spot $(50 \mathrm{kDa})$ was down-regulated at $26^{\circ} \mathrm{C}$ (Figure $1 \mathrm{~A}$ ) in comparison with exposure to $37^{\circ}$ $\mathrm{C}$ (Figure 1B). The $75 \mathrm{kDa}$ spot, which is upregulated at $26^{\circ} \mathrm{C}$, was identified by comparing spot pattern of $M$. 


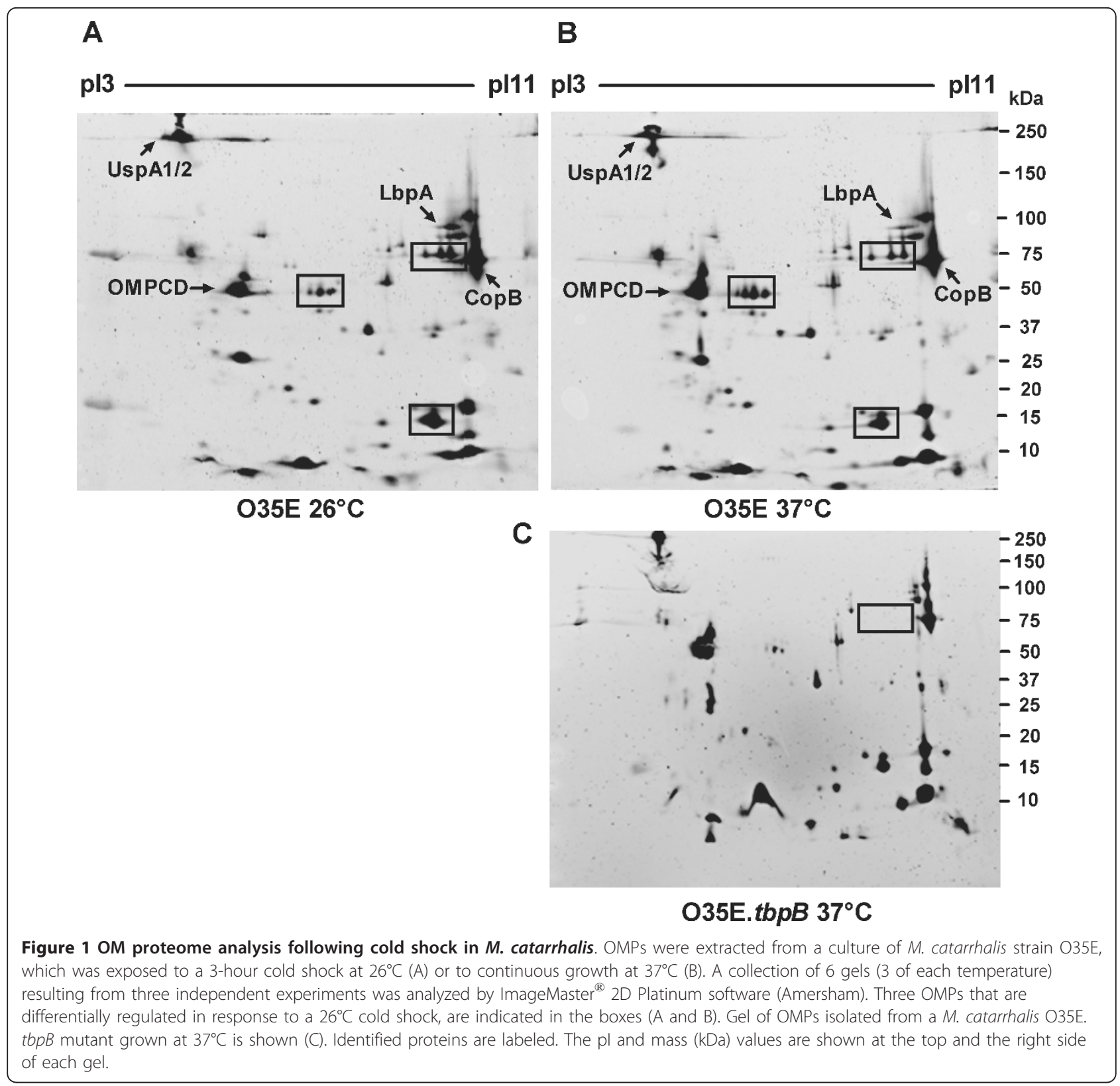

catarrhalis O35E wild-type and O35E.tbpB mutant strain as TbpB (Figure 1C), a peripheral OM lipoprotein possessing transferrin-binding properties, indicating that cold shock may increase iron acquisition, which is important for both growth and virulence.

Increased expression of genes involved in iron acquisition of $M$. catarrhalis induced by cold shock

To confirm the contribution of TbpB in the cold shock response, we assessed the $t b p B$ mRNA expression level of strain $\mathrm{O} 35 \mathrm{E}$ exposed to either $26^{\circ} \mathrm{C}$ or $37^{\circ} \mathrm{C}$. The expression level of $t b p B$ was significantly increased at $26^{\circ} \mathrm{C}$ in comparison to expression at $37^{\circ} \mathrm{C}$ (Figure $2 \mathrm{~A}$ ).
A similar expression pattern of $t b p B$ was also observed in $M$. catarrhalis clinical isolate 300 (data not shown). Cold shock at $26^{\circ} \mathrm{C}$ also enhanced the mRNA level of $t b p A$, an integral $\mathrm{OM}$ transferrin binding protein (Figure $2 \mathrm{~B})$. Low free iron conditions $(30 \mu \mathrm{M}$ of desferioxamine in the medium) caused an increase in gene transcription in bacteria grown at $37^{\circ} \mathrm{C}$ to a level similar to that seen in cells exposed to cold shock.

Because lactoferrin rather than transferrin is the primary carrier of iron on mucosal surfaces and lactoferrin binding proteins are thought to be important virulence factors in some gram-negative bacteria [28], we investigated whether cold shock affects the expression of these 
genes. As shown in Figure 2, cold shock increased the mRNA level of $l b p B$ and $l b p A$ genes in strain O35E after $3 \mathrm{~h}$ of incubation at $26^{\circ} \mathrm{C}$ (Figure 2C). Furthermore, cold shock increased the transcriptional level of $l b p A$ and $l b p B$ of other clinical isolates indicating that this effect is a general characteristic of $M$. catarrhalis (Figure 2D).

Enhanced binding of transferrin and lactoferrin on the surface of $M$. catarrhalis induced by cold shock

Because a temperature drop from $37^{\circ} \mathrm{C}$ to $26^{\circ} \mathrm{C}$ induces an increase in the copy numbers of genes involved in iron acquisition, we investigated whether it also affects the binding to human transferrin and lactoferrin. Strain O35E and its TbpB-deficient mutant were exposed to $26^{\circ} \mathrm{C}$ or $37^{\circ} \mathrm{C}$ and evaluated for their ability to bind transferrin. Binding to transferrin was increased when bacteria were exposed to $26^{\circ} \mathrm{C}$ (Figure 3A and 3B). The absence of $\mathrm{TbpB}$ reduced binding to transferrin, indicating that $\mathrm{TbpB}$ is required for maximum binding of transferrin on the surface of cold shock-induced $M$. catarhalis.

Binding to lactoferrin in a whole-cell solid-phase binding assay was significantly increased when bacteria were exposed to $26^{\circ} \mathrm{C}$, in comparison with exposure to $37^{\circ} \mathrm{C}$ (Figure 4A). The surface binding of human salivary and milk lactoferrin (sLf and Lf, respectively) was further quantitated using flow cytometry, resulting in a clear shift of fluorescence intensity for $M$. catarrhalis exposed at $26^{\circ} \mathrm{C}$ (Figure $4 \mathrm{~B}$ ). Immunoblot analysis revealed that OMPs isolated from strain $\mathrm{O} 35 \mathrm{E}$ exposed to $26^{\circ} \mathrm{C}$ display a greater lactoferrin binding capacity in comparison to OMPs isolated from cells exposed to $37^{\circ} \mathrm{C}$ (Figure 4C). The band with a strong lactoferrin-binding capacity and an apparent molecular weight of $100 \mathrm{kDa}$ most likely represents LbpA because only LbpA (103 kDa), an

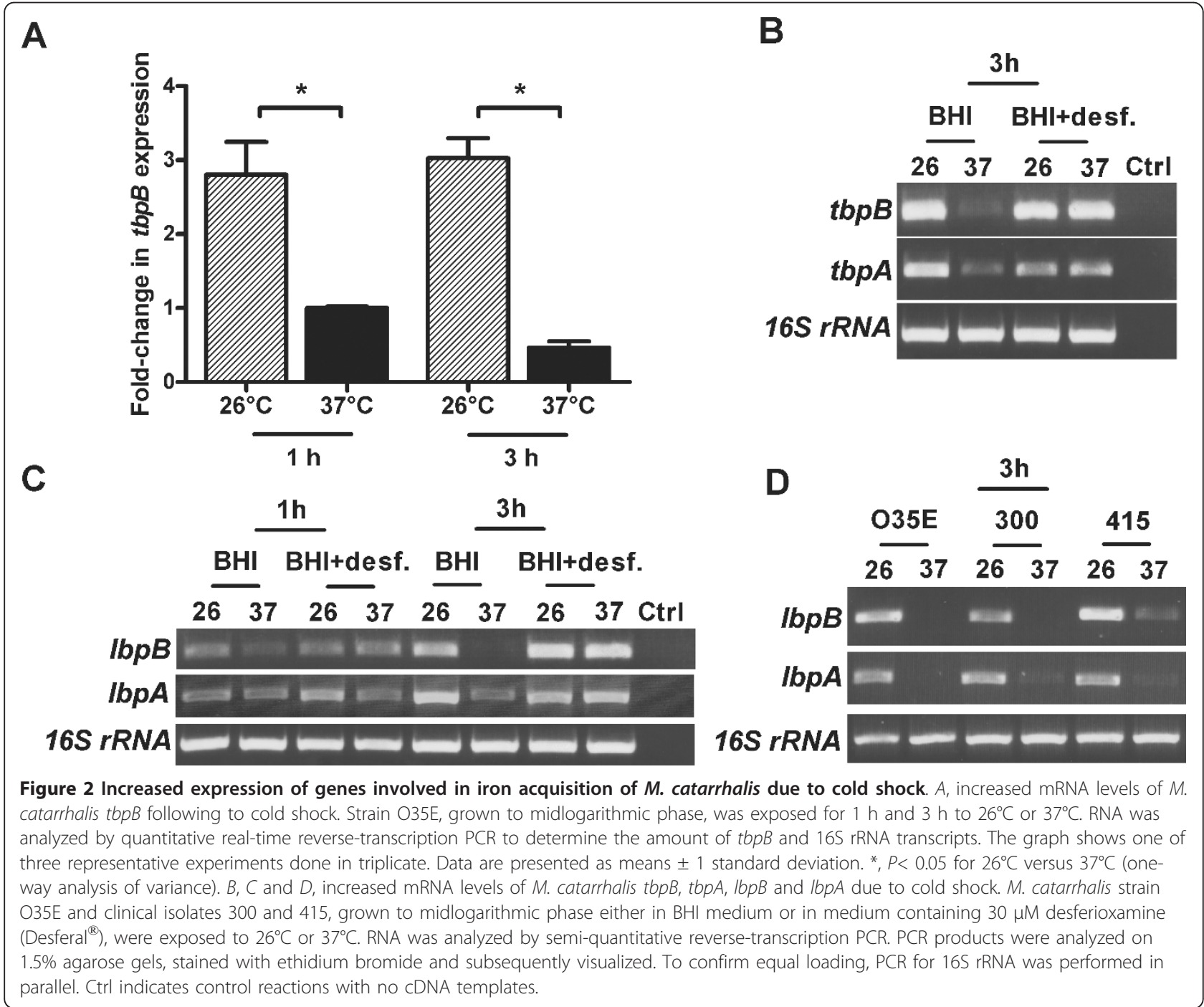




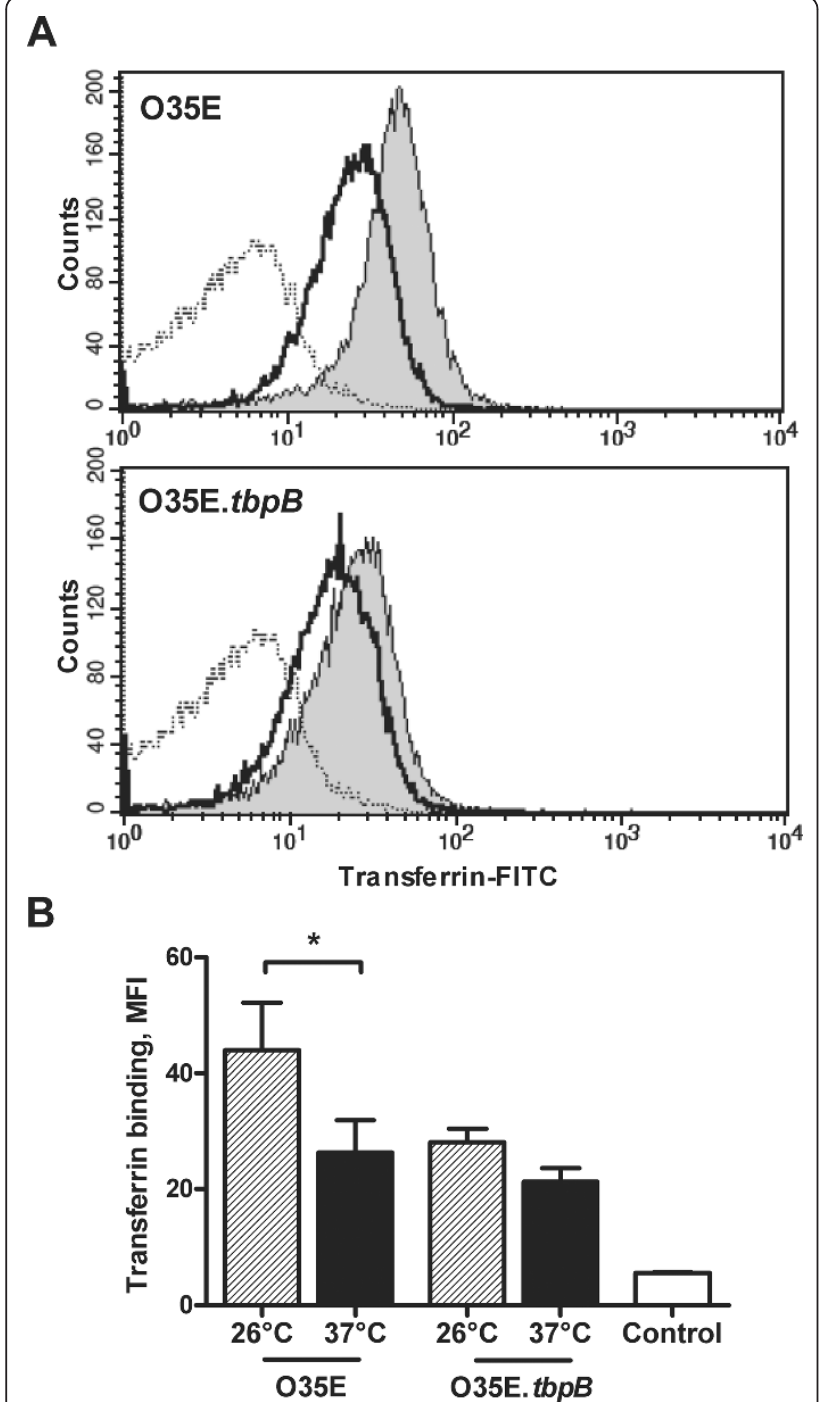

Figure 3 Increase in the binding of transferrin on the surface of $\boldsymbol{M}$. catarrhalis as a result of cold shock. A, strain O35E and its isogenic mutant $\mathrm{O} 35 \mathrm{E} . t b p \mathrm{~B}$ exposed to $26^{\circ} \mathrm{C}$ or $37^{\circ} \mathrm{C}$ for $3 \mathrm{~h}$ were incubated with fluorescein isothiocyanate (FITC)-conjugated transferrin $(0.1 \mu \mathrm{g} / \mathrm{mL})$ and flow cytometry analysis was performed. Shown are representative flow cytometry profiles of strain O35E and O35E.tbpB after exposure at $26^{\circ} \mathrm{C}$ (gray) or at $37^{\circ} \mathrm{C}$ (black), which demonstrate that $\mathrm{TbpB}$ is required for maximum binding of transferrin on the surface of cold shock-induced Moraxella catarrhalis. The dotted line represents the negative control (bacteria only). The mean fluorescence intensity \pm 1 standard deviation for three experiments performed is shown in panel $B .{ }^{*}, P<0.05$ for $26^{\circ}$ $\mathrm{C}$ versus $37^{\circ} \mathrm{C}$ (one-way analysis of variance)

integral OMP, is able to bind lactoferrin and is essential for iron acquisition from lactoferrin, whereas $\mathrm{LbpB}$ only plays a facilitating role [24].

Since lactoferrin is an antibacterial protein found in human secretions [26], it was important to determine its bactericidal effect on $M$. catarrhalis. No bactericidal effect was observed when $M$. catarrhalis strain $\mathrm{O} 35 \mathrm{E}$ was incubated with human lactoferrin (data not shown).

Because CopB is involved in the ability of M.catarrhalis to acquire iron from human lactoferrin and transferrin, we assessed the expression of this protein following cold shock. Flow cytometry analysis demonstrates that exposure of M.catarrhalis strain $\mathrm{O} 35 \mathrm{E}$ to $26^{\circ} \mathrm{C}$ increases the expression of $\mathrm{CopB}$ on the bacterial surface (Figure $4 \mathrm{D}$ and $4 \mathrm{E})$.

\section{Cold shock results in upregulation of UspA2 and} increases the binding of vitronectin on the surface of $M$. catarrhalis

To investigate the involvement of UspA2 in the cold shock response, we assessed uspA2 mRNA expression levels after exposure of $M$. catarrhalis to $26^{\circ} \mathrm{C}$ or $37^{\circ} \mathrm{C}$. Quantitative RT-PCR showed no significant differences between $26^{\circ} \mathrm{C}$ and $37^{\circ} \mathrm{C}$ with respect to the relative amount of uspA2 mRNA (data not shown). We determined the expression of UspA2 after cold shock on the surface of $M$. catarrhalis. Because the monoclonal antibody 17C7 recognizes both UspA1/A2, we used UspA1 and UspA2 mutants, respectively, of strain O35E. Expression of both UspA1 and UspA2 were increased on the surface of $M$. catarrhalis after cold shock (Figure $5 \mathrm{~A}$ and 5B). UspA2 mediates serum resistance of $M$. catarrhalis by binding vitronectin. Given that cold shock induces UspA2 expression, we hypothesized that a temperature downshift might increase surface binding of vitronectin. We preincubated $M$. catarrhalis grown at $26^{\circ} \mathrm{C}$ or $37^{\circ} \mathrm{C}$ with human vitronectin and determined vitronectin binding by flow cytomertry. Binding to vitronectin was increased when bacteria were exposed to $26^{\circ}$ $\mathrm{C}$ (Figure 5C and 5D). The absence of UspA2 diminished binding of vitronectin but did not abolish it, possibly due to UspA1 interactions with vitronectin [29]. Serum bactericidal assay with $M$. catarrhalis strain O35E exposed to $26^{\circ} \mathrm{C}$ or $37^{\circ} \mathrm{C}$ demonstrated that cold shock did not influence serum resistance of O35E strain (data not shown).

\section{Cold shock influences hag expression and binding of human IgD on the surface of $M$. catarrhalis}

To investigate the contribution of Hag to the cold shock response, we assessed the hag mRNA expression level of strain $\mathrm{O} 35 \mathrm{E}$ exposed to either $26^{\circ} \mathrm{C}$ or $37 \mathrm{C}$. The expression level of hag was significantly reduced at $26^{\circ} \mathrm{C}$ in comparison to expression at $37^{\circ} \mathrm{C}$ (Figure $6 \mathrm{~A})$. Addressing the question whether a decreased mRNA copy number of hag at $26^{\circ} \mathrm{C}$ translates into decreased expression of Hag on the bacterial surface, we performed immunoblot analysis with OMPs preparations of strains $\mathrm{O} 35 \mathrm{E}$ and 300 exposed at $26^{\circ} \mathrm{C}$ or $37^{\circ} \mathrm{C}$ for $3 \mathrm{~h}$ using human salivary IgA antibodies 


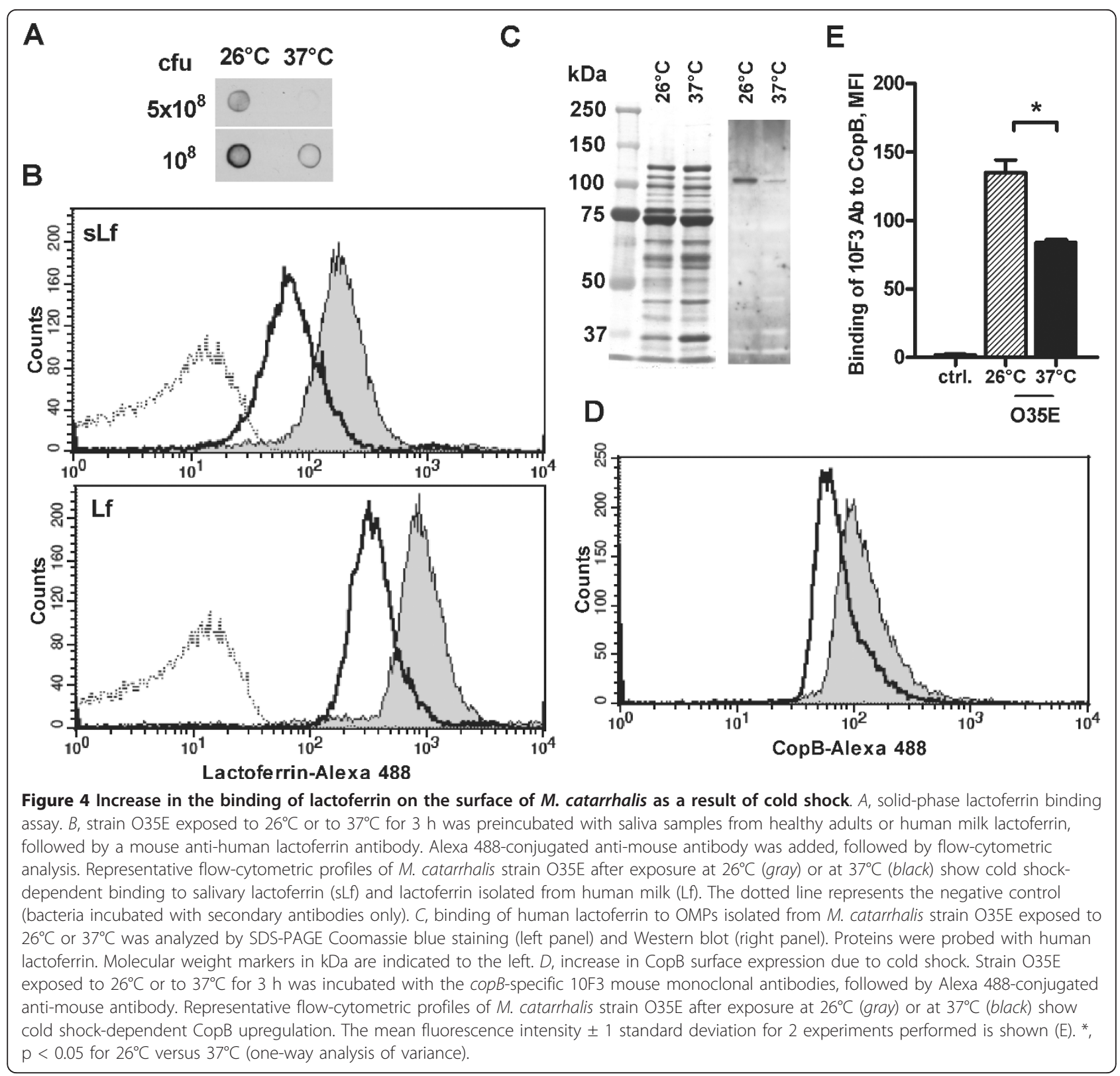

which specifically recognize surface exposed OMPs, including Hag [20]. Immunoblot analysis revealed that M. catarrhalis strains $\mathrm{O} 35 \mathrm{E}$ and 300 exposed at $26^{\circ} \mathrm{C}$ expressed smaller amounts of Hag protein compared to bacteria incubated at $37^{\circ} \mathrm{C}$ (Figure 6B). The Hagdeficient O35E.hag strain did not bind the Hag-specific salivary IgA (data not shown). Since Hag has been found to be responsible for $M$. catarrhalis binding to $\operatorname{IgD}$, we investigated IgD-binding on the surface of bacteria grown at $26^{\circ} \mathrm{C}$ or $37^{\circ} \mathrm{C}$. Flow cytometric analysis demonstrated a slight decrease of IgD-binding on the surface of strain O35E after cold shock, while IgDbinding to clinical isolate 300 was clearly reduced at $26^{\circ} \mathrm{C}$ (Figure 6C and 6D). The Hag-deficient mutant displayed an overall reduced IgD-binding level with increased binding of $\operatorname{IgD}$ at $26^{\circ} \mathrm{C}$ in comparison to $37^{\circ}$ $\mathrm{C}$, suggesting that other $\mathrm{OM}$ components might antagonize the Hag-mediated IgD-binding following cold shock. This concept is supported by previous findings demonstrating the ability of mucosal IgD to recognize lipopolysaccaride, a key cell wall component of gramnegative bacteria [30]. Indeed, the LOS-deficient mutant of $M$. catarrhalis strain O35E exhibited significantly decreased binding of $\operatorname{IgD}$ on the surface of cold shock-induced bacteria in comparison with exposure to $37^{\circ} \mathrm{C}$ (Figure $6 \mathrm{C}$ and $6 \mathrm{D}$ ). 


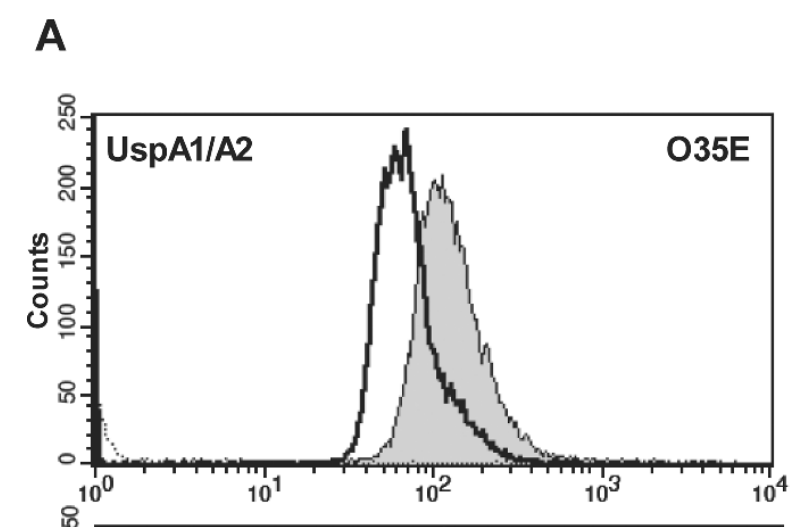

\section{C}


Figure 5 Cold shock results in upregulation of UspA2 and increases the binding of vitronectin on the surface of $M$. catarrhalis Representative flow-cytometric profiles of $\mathrm{M}$. catarrhalis strains O35E, O35E.uspA1 and O35E.uspA2 after exposure at $26^{\circ} \mathrm{C}$ (gray) or at $37^{\circ} \mathrm{C}$ (black) show cold shock-dependent UspA1/A2 upregulation (A) and UspA2-dependent binding to vitronectin (C). The dotted line represents the negative control (bacteria incubated with secondary antibodies only). The mean fluorescence intensity \pm 1 standard deviation for 2 experiments performed is shown (B and D). ${ }^{*}, \mathrm{p}<0.05$ for $26^{\circ} \mathrm{C}$ versus $37^{\circ} \mathrm{C}$ (one-way analysis of variance). 


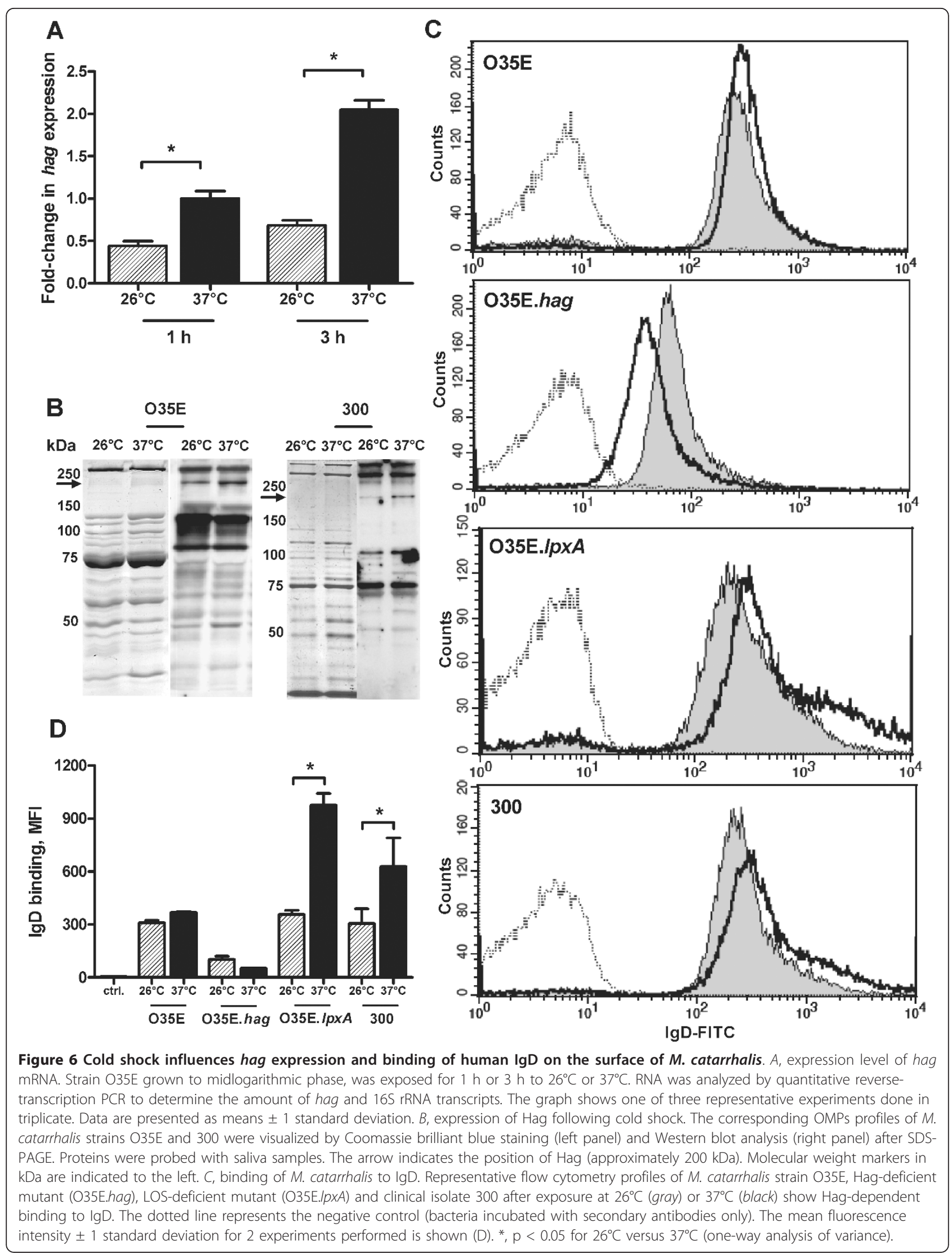




\section{Discussion}

In this study, we have analyzed the cold shock-induced changes in the OM proteome of $M$. catarrhalis and identified $\mathrm{TbpB}$, whose expression was increased more than two-fold after a $26^{\circ} \mathrm{C}$ cold shock, as a member of the iron acquisition systems that is important for both growth and virulence. Our data demonstrate that the expression of transferrin receptors and transferrin binding on the bacterial surface were also increased when M. catarrhalis was exposed to a $26^{\circ} \mathrm{C}$ cold shock. Transferrin is predominantly found in serum and in serous exudates. During pronounced inflammation, it is likely that the local tissue damage results in the transsudation of various iron sources, including transferrin, to mucosal surfaces acting as additional iron sources for $M$. catarrhalis [31].

We also demonstrate that a $26^{\circ} \mathrm{C}$ cold shock increased the expression of genes involved in lactoferrin acquisition and enhanced lactoferrin binding on the surface of $M$. catarrhalis. The overall presence of lactoferrin receptors in $M$. catarrhalis isolates suggests its important role in colonization or infection [32]. In our previous study we demonstrated that exposure of M. catarrhalis to $26^{\circ} \mathrm{C}$ increases the release of proinflammatory cytokine IL-8 in pharyngeal epithelial cells likely leading to the increased inflammation [10]. Thus, greater local concentrations of IL-8 would promote enhanced recruitment and influx of neutrophils that release lactoferrin from their secondary granules, which contribute to lactoferrin levels both locally and in the circulation [33,34]. On the other hand, increased expression of $M$. catarrhalis lactoferrin binding proteins following cold shock would facilitate the binding and acquisition of iron from lactoferrin to support growth of bacteria in the mucosal environment. It has been shown that supplemental lactoferrin can enhance the virulence of meningococcal infection in mice [35]. In addition to iron acquisition, lactoferrin receptors may provide protection against anti-bacterial cationic peptides (eg, lactoferricin) and reduce complement-mediated killing. The pneumococcal surface protein PspA binds lactoferrin and protects Streptococcus pneumoniae against the antibacterial effect of lactoferricin [26]. The release of LbpB from the cell surface by a NalP protease protects Neisseria meningitidis against bactericidal antibodies [36]. Therefore, increased expression of lactoferrin receptors and enhanced binding of lactoferrin on the surface of bacteria following cold shock might be associated with enhanced protection of M. catarrhalis against anti-bacterial cationic peptides and bactericidal antibodies.

The level of UspA2 protein that afforded serum resistance in the bactericidal activity assay has been shown to correlate with increased binding of vitronectin [37]. Our results indicate that cold shock upregulates the
UspA2 protein expression and promotes $M$. catarrhalis binding to vitronectin. Increased UspA2 protein expression at $26^{\circ} \mathrm{C}$ was not the result of higher copy number of uspA2 mRNA, indicating that post-transciptional mechanisms are involved in upregulation of this protein after cold shock [38]. Cold shock did not influence the serum resistance of $\mathrm{O} 35 \mathrm{E}$ strain indicating that $M$. catarrhalis strains may need to maintain a certain threshold level of UspA2 protein necessary to evade host defenses. Most seroresistant M. catarrhalis strains express at $37^{\circ} \mathrm{C}$ sufficient levels of UspA2 to mediate serum resistance [37]. It is conceivable that cold shock would increase UspA2 expression and vitronectin binding in $M$. catarrhalis strains constitutively expressing low levels of UspA2, leading to the enhanced serum resistance.

The infant population during the first year of life possesses a substantial proportion of IgD in saliva [39]. The interaction between Hag and IgD mediates B cell endocytosis and killing of $M$. catarrhalis whereas non-IgDbinding bacteria were not taken up by B cells [27]. Furthermore, IgD-stimulated mucosal basophils release antimicrobial factors inhibiting the replication of M. catarrhalis [30]. Here we demonstrate that cold shock at $26^{\circ} \mathrm{C}$ reduces the mRNA expression level of hag, Hag protein expression and the Hag-mediated binding of human IgD to the surface of $M$. catarrhalis. Decreased copy numbers of hag at $26^{\circ} \mathrm{C}$ were also found in other clinical isolates indicating that this effect is a general characteristic of seroresistant M. catarrhalis [9]. Therefore, reduced expression of Hag and decreased binding of IgD on the bacterial surface following cold shock might lead to reduced stimulation of $B$ cells and increased survival by prevention of endocytosis by these cells as well as to decreased stimulation of basophils leading to reduced release of antimicrobal factors. However, the presence of specific IgD against LOS triggered increased recognition of bacteria following cold shock (Figure 6). Consequently, children who lack LOS-specific IgD may be more susceptible to $M$. catarrhalis infections, particularly after exposure to cold air.

Three OMPs were found to be differentially (a greater than two fold change) regulated in response to a $26^{\circ} \mathrm{C}$ cold shock (Figure 1), while immunoblot and flow cytometric analysis revealed that several other OMPs are also involved in cold shock response. The lack of some differentially regulated OMPs in the 2-DE pattern might be the result of difficult identification or low abundance. Furthermore, protein spots with a fold change below the indicated threshold were considered by the Image Master $2 \mathrm{D}$ program as not relevant.

Thus, cold shock, which occurs when humans breathe cold air [7], is a physiologic phenomenon during the 
cold season and entails a range of adaptive events in the residential upper respiratory tract flora that lead to the stimulation of nutrient (e.g., iron)-acquistion, serum resistance and immune evasion potentially resulting in increased bacterial density on the nasopharyngeal surface. Clinical studies in children have demonstrated that the density of $M$. catarrhalis in the nasopharynx is positively associated with prolonged respiratory tract symptoms and a greater likelihood of purulent otitis media $[40,41]$. This study demonstrates that a $26^{\circ} \mathrm{C}$ cold shock induces the expression of genes involved in transferrin and lactoferrin acquisition, and enhances binding of these proteins on the surface of $M$. catarrhalis. Exposure of $M$. catarrhalis to $26^{\circ} \mathrm{C}$ upregulates both CopB and UspA2 expression, the latter leading to improved vitronectin binding on the surface of bacteria. In contrast, cold shock decreases the expression of Hag and reduces the IgD-binding on the surface of $M$. catarrhalis. These findings indicate that cold air in the human upper respiratory tract induces in $M$. catarrhalis a complex of adaptive mechanisms that may enable the bacterium to target their host cellular receptors or soluble effectors and consequently to display enhanced growth, colonization and virulence.

\section{Conclusions}

A physiologic cold shock as it occurs when humans breathe cold air for prolonged periods of time increases the capacity of $M$. catarrhalis for iron uptake from human lactoferrin and transferrin, enhances the capacity of $M$. catarrhalis to bind vitronectin, which neutralizes the lethal effect of human complement, and decreases IgD-binding by hemagglutinin. These data support the notion that $M$. catarrhalis uses physiologic exposure to cold air to upregulate pivotal survival systems in the human pharynx that may contribute to bacterial virulence. Thus, cold shock may exert adaptive events in at least one member of the residential upper respiratory tract flora of facultative pathogens, which may increase the bacterial density on the respiratory tract mucosal surface (which in turn is associated with an increased likelihood of acute otitis media).

\footnotetext{
Acknowledgements

This work was supported by the Swiss National Science Foundation (SNF) grants 3100A0-102246 and 3100A0-116053 (to CA). The authors thank Dr. Eric Hansen, University of Texas Southwestern Medical Center, Dallas, TX, for the kind gift of the monoclonal antibodies mAb10F3 and mAb17C7.
}

\section{Author details}

${ }^{1}$ Institute for Infectious Diseases, University of Bern, CH-3010 Bern, Switzerland. ${ }^{2}$ Division of Human Molecular Genetics, University of Bern, Inselspital, CH-3010 Bern, Switzerland. ${ }^{3}$ Department of Pediatrics, University of Bern, Inselspital, $\mathrm{CH}-3010$ Bern, Switzerland.

\section{Authors' contributions}

VS conceived of the study, designed the experiments, conducted the majority of the experimental work and wrote the manuscript. RT performed the comparative SDS-PAGE analyses. AS performed and analyzed the 2-DE and MALDI-TOF experiments. CA conceived the study, designed the experiments and finalized the manuscript. All authors read and approved the final manuscript.

Received: 8 April 2011 Accepted: 12 August 2011

Published: 12 August 2011

\section{References}

1. Faden H, Duffy R, Wasielewski R, Wolf J, Krystofik D, Tung Y: Relationship between nasopharyngeal colonization and the development of otitis media in children. J Infect Dis 1997, 175:1440-5.

2. Palmu A, Herva E, Savolainen H, Karma P, Mäkela PH, Kilpi T: Association of clinical signs and symptoms with bacterial findings in acute otitis media. Clin Infect Dis 2004, 38:234-42.

3. Van Hare GF, Shurin PA: The increasing importance of Branhamella catarrhalis in respiratory infections. Pediatr Infect Dis J 1987, 6:92-4.

4. Mbaki N, Rikitomi N, Nagatake T, Matsumoto K: Correlation between Branhamella catarrhalis adherence to oropharyngeal cells and seasonal incidence of lower respiratory tract infections. Tohoku J Exp Med 1987, 153:111-21.

5. Sarubbi FA, Myers JW, Williams JJ, Shell CG: Respiratory infections caused by Branhamella catarrhalis. Selected epidemiologic features. Am J Med 1990, 88:9-14.

6. Hendley JO, Hayden FG, Winther B: Weekly point prevalence of Streptococcus pneumoniae, Hemophilus influenzae and Moraxella catarrhalis in the upper airways of normal young children: effect of respiratory illness and season. APMIS 2005, 113:213-20.

7. Rouadi P, Baroody FM, Abbott D, Naureckas E, Solway J, Naclerio RM: A technique to measure the ability of the human nose to warm and humidify air. J Appl Physiol 1999, 87:400-6.

8. Sun K, Metzger DW: Inhibition of pulmonary antibacterial defense by interferon-gamma during recovery from influenza infection. Nat Med 2008, 14:558-64.

9. Heiniger N, Troller R, Meier PS, Aebi C: Cold shock response of the UspA1 outer membrane adhesin of Moraxella catarrhalis. Infect Immun 2005, 73:8247-55

10. Spaniol V, Troller R, Aebi C: Physiologic cold shock increases adherence of Moraxella catarrhalis to and secretion of interleukin 8 in human upper respiratory tract epithelial cells. J Infect Dis 2009, 200:1593-601.

11. Jetter M, Spaniol V, Troller R, Aebi C: Down-regulation of porin M35 in Moraxella catarrhalis by aminopenicillins and environmental factors and its potential contribution to the mechanism of resistance to aminopenicillins. J Antimicrob Chemother 2010, 65:2089-96.

12. Aebi C, Stone B, Beucher M, Cope LD, Maciver I, Thomas SE, McCracken GH Jr, Sparling PF, Hansen EJ: Expression of the CopB outer membrane protein by Moraxella catarrhalis is regulated by iron and affects iron acquisition from transferrin and lactoferrin. Infect Immun 1996, 64:2024-30.

13. Helminen ME, Maciver I, Latimer JL, Klesney-Tait J, Cope LD, Paris M, McCracken GH Jr, Hansen EJ: A large, antigenically conserved protein on the surface of Moraxella catarrhalis is a target for protective antibodies. $\mathrm{J}$ Infect Dis 1994, 170:867-72.

14. Attia AS, Ram S, Rice PA, Hansen EJ: Binding of vitronectin by the Moraxella catarrhalis UspA2 protein interferes with late stages of the complement cascade. Infect Immun 2006, 74:1597-611.

15. Nordstrom T, Blom AM, Tan TT, Forsgren A, Riesbeck K: lonic binding of C3 to the human pathogen Moraxella catarrhalis is a unique mechanism for combating innate immunity. J Immunol 2005, 175:3628-36.

16. Nordstrom T, Blom AM, Forsgren A, Riesbeck $\mathrm{K}$ : The emerging pathogen Moraxella catarrhalis interacts with complement inhibitor C4b binding protein through ubiquitous surface proteins A1 and A2. J Immunol 2004, 173:4598-606.

17. Pearson MM, Lafontaine ER, Wagner NJ, Geme III JW, Hansen EJ: A hag mutant of Moraxella catarrhalis strain O35E is deficient in hemagglutination, autoagglutination, and immunoglobulin D-binding Activities. Infect Immun 2002, 70:4523-33. 
18. Holm MM, Vanlerberg SL, Sledjeski DD, Lafontaine ER: The Hag protein of Moraxella catarrhalis strain O35E is associated with adherence to human lung and middle ear cells. Infect Immun 2003, 71:4977-84.

19. Gjorloff WA, Hadzic R, Forsgren A, Riesbeck K: The novel IgD binding protein from Moraxella catarrhalis induces human B lymphocyte activation and Ig secretion in the presence of Th2 cytokines. J Immunol 2002, 168:5582-8.

20. Stutzmann Meier P, Heiniger N, Troller R, Aebi C: Salivary antibodies directed against outer membrane proteins of Moraxella catarhalis in healthy adults. Infect Immun 2003, 71:6793-8.

21. Spaniol V, Heiniger N, Troller R, Aebi C: Outer membrane protein UspA1 and lipooligosaccharide are involved in invasion of human epithelial cells by Moraxella catarrhalis. Microbes Infect 2008, 10:3-11.

22. Heiniger N, Spaniol V, Troller R, Vischer M, Aebi C: A reservoir of Moraxella catarrhalis in human pharyngeal lymphoid tissue. J Infect Dis 2007, 196:1080-7.

23. Murphy TF, Loeb MR: Isolation of the outer membrane of Branhamella catarrhalis. Microb Pathog 1989, 6:159-74.

24. Bonnah RA, Wong $H$, Loosmore SM, Schryvers AB: Characterization of Moraxella (Branhamella) catarrhalis $l b p B, I b p A$, and lactoferrin receptor orf3 isogenic mutants. Infect Immun 1999, 67:1517-20.

25. Schaller A, Troller R, Molina D, Gallati S, Aebi C, Stutzmann Meier P: Rapid typing of Moraxella catarrhalis subpopulations based on outer membrane proteins using mass spectrometry. Proteomics 2006, 6:172-80

26. Shaper M, Hollingshead SK, Benjamin WH Jr, Briles DE: PspA protects Streptococcus pneumoniae from killing by apolactoferrin, and antibody to PspA enhances killing of pneumococci by apolactoferrin. Infect Immun 2004, 72:5031-40.

27. Vidakovics ML, Jendholm J, Mörgelin M, Månsson A, Larsson C, Cardell LO, Riesbeck K: B cell activation by outer membrane vesicles-a novel virulence mechanism. PLOS Pathog 2010, 6:e1000724.

28. Pettersson A, Prinz T, Umar A, van der Biezen J, Tommassen J: Molecular characterization of $\mathrm{LbpB}$, the second lactoferrin-binding protein of Neisseria meningitidis. Mol Microbiol 1998, 27:599-610.

29. McMichael JC, Fiske MJ, Fredenburg RA, Chakravarti DN, VanDerMeid KR, Barniak V, Caplan J, Bortell E, Baker S, Arumugham R, Chen D: Isolation and characterization of two proteins from Moraxella catarrhalis that bear a common epitope. Infect Immun 1998, 66:4374-81.

30. Chen K, Xu W, Wilson M, He B, Miller NW, Bengtén E, Edholm ES, Santini PA, Rath P, Chiu A, Cattalini M, Litzman J, B Bussel J, Huang B, Meini A, Riesbeck K, Cunningham-Rundles C, Plebani A, Cerutti A: Immunoglobulin $D$ enhances immune surveillance by activating antimicrobial, proinflammatory and B cell-stimulating programs in basophils. Nat Immunol 2009, 10:889-98.

31. Schryvers AB, Stojiljkovic I: Iron acquisition systems in the pathogenic Neisseria. Mol Microbiol 1999, 32:1117-23.

32. Ogunnariwo JA, Schryvers AB: Rapid identification and cloning of bacterial transferrin and lactoferrin receptor protein genes. J Bacteriol 1996, 178:7326-8.

33. Wellnitz O, Kerr DE: Cryopreserved bovine mammary cells to model epithelial response to infection. Vet Immunol Immunopathol 2004, 101:191-202

34. Juffrie M, van Der Meer GM, Hack CE, Haasnoot K, Sutaryo, Veerman AJ, Thijs LG: Inflammatory mediators in dengue virus infection in children: interleukin- 8 and its relationship to neutrophil degranulation. Infect Immun 2000, 68:702-7.

35. Schryvers AB, Gonzalez GC: Comparison of the abilities of different protein sources of iron to enhance Neisseria meningitidis infection in mice. Infect Immun 1989, 57:2425-9.

36. Roussel-Jazede V, Jongerius I, Bos MP, Tommassen J, van Ulsen P: NalPmediated proteolytic release of lactoferrin-binding protein $B$ from the meningococcal cell surface. Infect Immun 2010, 78:3083-9.

37. Attia AS, Hansen EJ: A conserved tetranucleotide repeat is necessary for wild-type expression of the Moraxella catarrhalis UspA2 protein. J Bacteriol 2006, 188:7840-52.

38. Gualerzi CO, Giuliodori AM, Pon CL: Transcriptional and posttranscriptional control of cold-shock genes. J Mol Biol 2003, 331:527-39.

39. Seidel BM, Schubert S, Schulze B, Borte M: Secretory IgA, free secretory component and IgD in saliva of newborn infants. Early Hum Dev 2001, 62:159-64.
40. Kristo A, Uhari M, Kontiokari T, Glumoff $\mathrm{V}$, Kaijalainen $T$, Leinonen $M$, Luotonen J, Koivunen P, Kujala T, Pokka T, Alho OP: Nasal middle meatal specimen bacteriology as a predictor of the course of acute respiratory infection in children. Pediatr Infect Dis J 2006, 25:108-12.

41. Smith-Vaughan $H$, Byun R, Nadkarni M, Jacques NA, Hunter N, Halpin S, Morris PS, Leach AJ: Measuring nasal bacterial load and its association with otitis media. BMC Ear Nose Throat Disord 2006, 6:10.

doi:10.1186/1471-2180-11-182

Cite this article as: Spaniol et al:: Physiologic cold shock of Moraxella catarrhalis affects the expression of genes involved in the iron acquisition, serum resistance and immune evasion. BMC Microbiology 2011 11:182.

\section{Submit your next manuscript to BioMed Central and take full advantage of:}

- Convenient online submission

- Thorough peer review

- No space constraints or color figure charges

- Immediate publication on acceptance

- Inclusion in PubMed, CAS, Scopus and Google Scholar

- Research which is freely available for redistribution

Submit your manuscript at www.biomedcentral.com/submit
Biomed Central 